\title{
Initial Experience with Endoscopic Ultrasound
}

\author{
Shil BC, Banik RK, Saha SK, Faruque MO, Islam ASMN, Rahman MH \\ Department of Gastroenterology, Sir Salimullah Medical College \& Mitford Hospital, Dhaka. \\ Email: bimalcshil@yahoo.com
}

\begin{abstract}
Since its introduction in early 1990s, endoscopic ultrasound (EUS) has become integral to the diagnosis and staging of various luminal, extraluminal gastrointestinal (GI) and certain non-GI lesions. There is no data on EUS experience in Bangladesh. The aim of this paper is to evaluate the initial recent experience and clinical impact of EUS. All EUS procedures data were recorded prospectively from July 2013 to December 2014. These included patients' demographics, referral details, provisional diagnosis, management plan before and after EUS \& indications of procedures. EUS-FNA data recorded included details regarding site, number of passes and histological diagnosis. Two hundred \& four EUS procedures were carried out over one and half years. Male female ratio was 1.4:1, mean age was $46.4 \pm 20$ years. Of these procedures $148(72.5 \%)$ were referrals from physicians and $56(27.5 \%)$ were from surgeons. Most common indications were pancreatobiliary pathologies, esophageal \& gastric pathologies. Pancreatobiliary lesions $(n=165,80.9 \%)$ included patients with (A) Benign pathologies: Microliths in Gall baldder $(n=6)$, Gall stones $(n=12)$, Biliary ascarrisis $(n=22)$, Choledocholithiasis $(n=42)$, Acute Pancreatitis $(n=9)$, Chronic Pancreatitis $(n=15)$, Pancreatic pseudocysts(n=4) (B) Malignant Pathologies : GB Carcinoma(n=4), Cholangiocarcinoma ( $n=29), C a-$ pancreas $(n=9)$, Periampullary carcinoma $(n=12)$. Esophageal lesion was $9.3 \%(n=19)$ of total procedures. Forty seven percent $(n=9)$ of EUS procedures on esophagus were for staging of esophageal malignancy, $10.5 \%(n=2)$ for restaging or recurrence after chemoradiation and $21 \%(n=4)$ for submucosal lesions. Fifteen EUS procedures were carried out for gastric lesions, 07 were for staging of gastric carcinoma, 04 were for assessment of submucosal lesions (e.g. GIST, lipoma or external compression), 02 for assessment of polyps and 02 for gastric ulcers.In clinical impact \& outcome study, changes in diagnosis, management, avoidance of investigations and usefulness of EUS were evaluated. Diagnosis was changed in $34.4 \%$ (64/186) \& management was changed in 45\%(92/204). Additional investigation was avoided in $57.8 \%$ (118/204). This is the first report of Bangladesh experience of EUS to date. EUS is safe, accurate, cost effective \& very useful tool for diagnosis and management of G.I. disorders.
\end{abstract}

\section{Introduction}

Endoscopic ultrasound (EUS) is the only method that combines conventional endoscopic viewing of Gastrointestinal (G.I.) lumen with ultrasound imaging of gut wall in fine detail and surrounding structures $^{1,2}$. It has been shown to be very useful in the evaluation of submucosal lesion, neuroendocrive tumors, staging of oesophagogastric tumors and pancreatobiliary lesions ${ }^{3}$. Standard EUS is performed with echoendoscope with ultrasound transducer mounted at the tip of the endoscope. There are two types of echoendoscopes for imaging: radial \& linear. The radial EUS produces imaging perpendicular to scope shaft and view like a CT scan and the linear EUS provides ultrasound images in a plane parallel to the direction of the insertion of the echoendoscope. The linear echoendoscope can be utilized to perform fine needle aspiration (FNA) or core biopsy and in the treatment of a variety of clinical condition. ${ }^{1,2,4,5}$
Since its introduction, EUS and EUS-FNA have become integral to the diagnosis and staging of various luminal, extra luminal GI and certain nonG.I. lesions. ${ }^{6,7}$ Therapeutic applications of EUS are rapidly expanding and facilitating various interventions like celiac plexus block or neurolysis, pancreatic collection or pseudo cyst drainage etc. ${ }^{1,4}$ There is no data on EUS experience in Bangladesh. Still we have only few centers with EUS facility. The aim of this paper is to evaluate the initial recent experience and clinical implications of EUS.

\section{Materials and Methods}

Sir Salimullah Medical College and Mitford Hospital is a large tertiary hospital situated at Dhaka city. This is the only active EUS centre in government sector, so it has a countrywide referral base.

EUS was performed using Fujinon SU-8000 EUS system, EG-530UR2 and EG-530UT2 radial and linear echoendoscopes (Fujifilm Inc., Japan). The 
scopes have transducer with variable frequency (5.0, 7.5, 10.0, 12.0 MHz). FNA was performed by 22 gauze needle (Boston-scientific). Patients having any of the following criteria were excluded from the study:

(i) acute abdomen;

(ii) poor general condition;

(iii) serious hepatic dysfunction;

(iv) severe co morbidity-respiratory or cardiac disorder

(v) pregnancy or possible pregnancy

The patients were on conscious sedation using I/V midazolam and fentanyl with or without ketamine. This was prospective analysis of 204 consecutive patients referred for UGIT-EUS over a period of one and half years from July 2013 to December 2014. Data collected were

i) Patients' demographics and referral details

ii) Provisional diagnosis and management plan before EUS

iii) Indication for EUS

iv) Anatomical sites evaluated

v) Echofeature of lesion

vi) EUS diagnosis

vii) Sedation used

viii)Technical difficulties and complication and

ix) Post EUS management plan

EUS-FNA data recorded included details regarding site, number of passes and histological diagnosis. Most of procedures were performed as outpatient basis unless patients were in inpatients or planned for FNA. Patient's informed consent was taken before EUS. Prospective clinical impact, outcome study and accuracy were evaluated.

\section{Results}

A total of 204 patients were examined over one and half years, male patients comprised $55.4 \%(n=113)$. The mean age of the patients was 46.4 years (age range 16 to 76 years). There has been a steady increase in the number of EUS performed day by day since the introduction of EUS in our department (shown in figure -1).

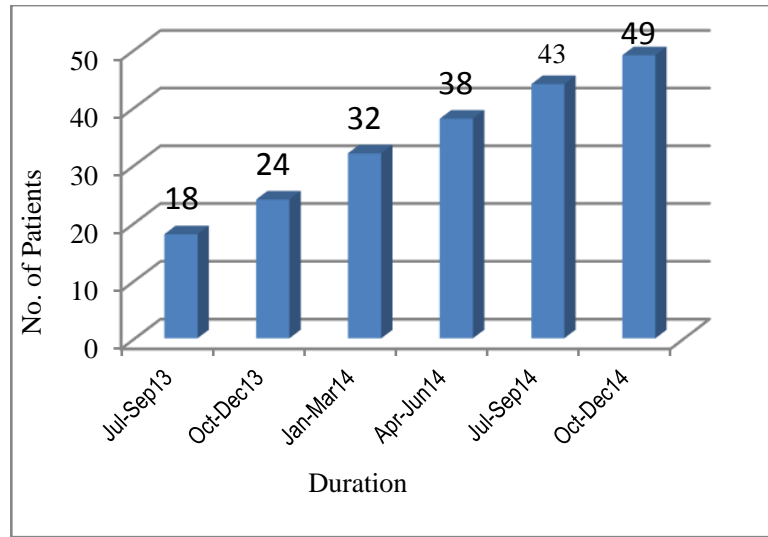

Fig.1 showing steady increase of EUS patients day by day
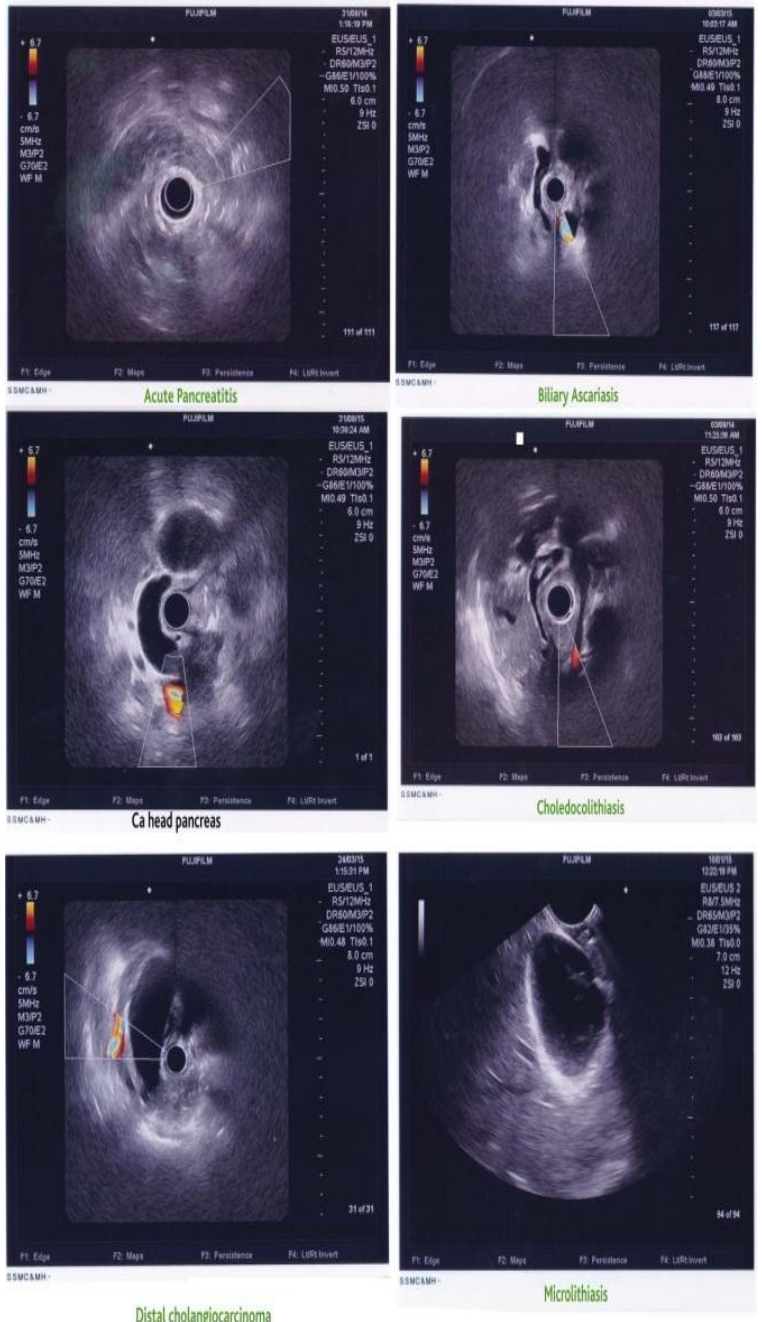

Fig.2: Endosonography images

Referrals: Most of the patients were referred from within SSMC and Mitford Hospital or from other hospitals of Dhaka division $(151,74 \%)$. The rest of the patients were from other division such as Chittagong (18, 8.8\%), Sylhet (22, 10.78\%), Barisal (6, 2.94\%), Rajshahi (4, 1.96\%), Khulna (3, $1.47 \%)$. Among the total procedure 148 (72.5\%) were from physicians and $56(27.5 \%)$ were from surgeons. Of the referrals from physicians, 107 $(72.29 \%)$ were from gastroenterologists and 28 (18.9\%) from internists and rest from hepatologists, chest physicians and oncologists etc. Among surgical referrals $44(78.6 \%$ ) were from the general surgeons and $12(21.4 \%)$ from thoracic surgeons.

Indications: Most common indications were pancreatobiliary lesions, esophageal and gastric lesions and less common indications were duodenal and mediastinal lesions.

Pancreatobiliary lesions: Indications were to assess pancreas, extrahepatic and intrahapatic bile ducts, gallbladder and ampullary or periampullary lesions. Frequency of lesions is shown in Table I. 
Table I: Showing frequency of pancreatobiliary lesions $(\mathrm{n}=169$, $80.9 \%$ )

\begin{tabular}{lc}
\hline Indications & No.of patients (\%) \\
\hline A) Benign pathologies & \\
Microliths in gall bladder & $06(3.63)$ \\
Cholelithiasis & $12(7.27)$ \\
Biliary sludge & $08(4.84)$ \\
Choledocholithiasis & $42(25.45)$ \\
Biliary ascariasis & $22(13.33)$ \\
Acute pancreatitis & $09(5.54)$ \\
Chronic pancreatitis & $15(9.09)$ \\
Pancreatic pseudocyst & $04(2.42)$ \\
B) Malignant pathologies & \\
Ca gall bladder & $04(2.42)$ \\
Cholangiocarcinoma & $29(17.57)$ \\
Periampullary carcinoma & $12(7.27)$ \\
Ca pancreas & $09(5.54)$ \\
\hline
\end{tabular}

Some patients had dual pathologies.

Esophageal lesions: Most common indication for assessment of esophageal disease was for TNM staging of malignancy. Frequency of esophageal lesions is shown in Table II.

Gastric lesions: Frequency of Gastric lesions is shown in Table II.

Duodenal lesions : Only 03 cases were to assess the lesions. Two cases were polyps and one was duodenal carcinoma.

Sedation: Conscious sedation was achieved to perform the procedure in all patients. All patients received fentanyl $(40 \pm 10 \mathrm{umg}$; mean \pm SD), midazolam was used in $30 \%$ cases with dosage of $2.5 \pm 1 \mathrm{mg}$; mean $\pm \mathrm{SD}$ ), ketamine was used in few cases (9 cases) only. There was no complication during or after sedation used.

EUS- FNA: EUS guided FNA was carried out in seven cases only. The frequency is shown in Table II.

Table II showing frequency of esophageal lesions, gastric lesions \& frequency of EUS FNA:

\begin{tabular}{lc}
\hline Esophageal Indications (n=19, 9.3\%), & $\begin{array}{c}\text { No. of } \\
\text { patients (\%) }\end{array}$ \\
\hline Staging of Ca-esophagus & $09(47.3 \%)$ \\
Restaging of Ca-esophagus after chemoradiation & $02(10.52 \%)$ \\
Submucosal lesion & $04(21.05 \%)$ \\
External compression & $02(10.52 \%)$ \\
Achalasia or pseudoachalasia & $01(5.26 \%)$ \\
Assessment of stricture & $01(5.26 \%)$ \\
\hline \multicolumn{1}{c}{ Frequency of Gastric lesions $(\mathrm{n}=15,7.4 \%)$} \\
\hline Indications & No. of \\
& patients (\%) \\
\hline Staging of Ca-stomach & $07(46.66 \%)$ \\
Submucosal lesion & $04(26.66 \%)$ \\
Polyps & $02(3.33 \%)$ \\
Gastric Ulcers & $02(13.33 \%)$ \\
\hline Frequency of EUS FNA: & No. of \\
\hline Indications (n=7) & patients (\%) \\
\hline Pancreatic mass (3) & $3(42.85 \%)$ \\
Submucosal lesion (3) & $3(42.85 \%)$ \\
Medestinal lesion/lymphnode (1) & $1(14.28 \%)$ \\
\hline
\end{tabular}

Clinical Implication and outcome study: EUS resulted in change of diagnosis in $34.4 \%$ (64/186) and management was altered in $45 \%(92 / 204)$. Additional investigations were considered unnecessary in $57.8 \%(118 / 204)$ but for evaluation of pancreatic duct in chronic pancreatitis and hilar lesion in biliary tree, further imaging were needed. The overall accuracy of endosonography was $72.5 \%$ (29/40) in our study when histological report was available. It has an excellent concordance $>82 \%$ with other imaging tests like CT, MRI \& ERCP but has clear superiority over conventional US in particular situations (e.g. pancreatobiliary lesions)

EUS was considered to be very useful according to referring doctors and they will continue to an EUS again to manage the similar patients.

Most useful situations: EUS Assessment of common bile duct lesions (e.g. unexplained dilatation of CBD, biliary ascariasis, CBD stone etc) were very useful, avoiding additional imaging in $96 \%$ of cases. It could avoid ERCP or surgery in $40 \%$ of cases. In submucosal lesions of esophagus and stomach, EUS played important role to avoid surgery (52\%).

Complications:_No complications were seen in this series.

\section{Discussion}

Our study shows that EUS is a safe and very useful tool for investigating different G.I. disorders. No procedure based or sedation related complication was seen. EUS is increasingly accepted by gastroenterologists, internists, surgeons and oncologists in our country as is shown by the increasing demand day by day. The current study shows that most of the referrals were within our hospital and from Dhaka division. Small numbers of cases were from other division of the country. The wide referral base suggests the need for the establishment of EUS units in all medical college hospitals of Bangladesh.

The population demographic data of this study reflects the heterogeneity of different G.I. lesions which may be benign to malignant. This reflects the importance of EUS in suspected G.I or non G.I pathologies. Our demographic data is comparable to that of Kalade et al and Kaffes at al. ${ }^{1,2}$

In our experience, most common referrals for EUS were from gastroenterologists (107, 52.45\%) followed by surgeons $(56,27.5 \%)$ and internists $(28,13.7 \%)$. This compares similarity to that of Australian study ${ }^{1}$. Majority of the EUS was carried out for pancreatobiliary indications, mostly for choledocholithiasis $(42,25.45 \%)$ followed by CCA $(29,17.57 \%)$ and biliary ascariasis $(22,13.33 \%)$. The pancreatobiliary lesions were common in study 
of Chong et $\mathrm{al}^{8}$ but esophageal indication was commonest in study of Kalade et al. ${ }^{1}$

Accurate diagnoses of pancreatobiliary disorders in patients of obstructive jaundice are important both for gastroenterologists and surgeons. Transabdominal ultrasonography has a low sensitivity $(50 \%$ to $70 \%)$ in identifying that etiologies of biliary abnormalities but EUS is very accurate in diagnosing CBD stone with an overall accuracy of $96 \%$ esp. with small calculi $(5 \mathrm{~mm})$ or calculi with non dilated biliary system. It also picks up small resectable pancreatobiliary mass as well as in diagnosing loco regional involvement with high sensitivity $(93-100 \%) .9,10,11$

Idiopathic acute pancreatitis (IAP) is a diagnostic challenge. Finding a treatable cause may help to prevent recurrent pancreatitis. EUS is a very useful modality to investigate IAP. ${ }^{12}$ Gall stones of size varying from as small as $2 \mathrm{~mm}$ to $5 \mathrm{~mm}$ and biliary sludge could be detected by EUS. We found gall bladder with microlithiasis in 6 cases of 10 IAP cases.

Esophageal indications $(19,9.3 \%)$ were the second highest in number in our study of which $57.8 \%$ was for assessment and TNM staging of ca-esophagus. Esophageal cancer is a devastating disease with a significant impact on patients' lives and health care systems worldwide. Staging of Ca-esophagus is extremely important since it helps to differentiate treatment options. For early disease, endoscopic mucosal resection (EMR) have shown a five year survival of $98 \%$ and a low recurrence rate ${ }^{13,14}$. CT and MRI lack the ability to differentiate layers of the esophageal wall. EUS is the most accurate to visualize the individual layers and this particularly useful in local staging including regional lymph node involvement ${ }^{1,13,14}$. Our all Ca-esophagus patients were referred from chest surgeons to see the possibility of curative surgery. So it emphasizes the usefulness of EUS in the management of such patients.

Submucosal tumor (SMT) is defined as intramural growth underneath the mucosa where etiology cannot readily be determined by endoscopy or barium study. EUS is the first choice for examining SMT due to high sensitivity and specificity. EUS is useful in providing information about origin, size, borders, echogenicity, anechoicity, vascularity, intramural or extraluminal impression of lesions. EUS can indicate whether endoscopic resection is possible or not ${ }^{15,16}$. In our study we found 04 cases of esophageal and 04 cases of gastric SMT.

The importance of tumor staging of $\mathrm{Ca}$ Stomach has been increasing with the development of endoscopic resection of the lesion. ${ }^{17}$ EUS has been shown to be accurate in staging of gastric cancers for both tumor and lymph nodes. ${ }^{2}$ Thereby EUS could influence the management by selecting the patients who will benefit from EMR, curative resection or palliative surgery. ${ }^{18} \mathrm{We}$ got 07 patients of Ca stomach in our study for staging. Staging was $85.7 \%$ perfect when compared with other imaging technique or surgery.

EUS guided FNA was carried out in 07 cases in our study. This is because in our early part of study we had only the radial scope, but for last 3 months we got the linear scope and FNA-Needle. In (5/7) $71.4 \%$ cases we got the accuracy in relation to histological diagnosis which is comparable with other study. ${ }^{1}$ Up to $45 \%$ patients had a significant change in management plan after endosonography which is well coincided with the finding of Kaffes et $\mathrm{al}^{2}$ and Kalade et al. ${ }^{1}$ Over all accuracy in diagnosis and staging of tumors were $72.5 \%$ in our series which is also comparable with the finding of Australian study. ${ }^{1,2}$

In our series, we had no major complication. Risk of radial EUS alone is comparable to UGI endoscopy. Complication rate of EUS-FNA is less than $0.1-1 \%$. $^{1,6}$

Large number of the referrals from gastroenterologists, hepatologists, internist, surgeons, chest physicians and surgeons and oncologists reflects the close interdepartmental cooperation and emphasizes multidisciplinary approach in patients care and management.

Conclusion: This is the first report of Bangladesh experience of EUS to date. EUS is safe, accurate, cost effective and very useful tool for diagnosis and management of G.I. disorders. We should develop our expertise and accessibility in this field with great emphasis for patients' sake.

\section{Conflict of Interest: None}

\section{Acknowledgements}

The authors would like to thank nurses and staffs of our department for their efforts in data acquisition, arrangement of patients follow up and assistance to perform EUS.

\section{References}

1. Kalade AV, Desmond PV, Chen RY. Experience of endoscopic ultrasound in an Australian tertiary hospital, ANZ J. Surg. 2006; 76: 1075-80.

2. Kaffes AJ, Mishra A, Simpson SB, Jones DB. Upper gastrointestinal endoscopic ultrasound and its impact on patient management: 1990-2000. Intern Med J 2002; 32: 372-78.

3. Jafri IH, Saltzman JR, Colby JM, Krims PE. Evaluation of the clinical impact of endoscopic ultrasonography in gastrointestinal disease. Gastrointest Endosc 1996; 44(4): $367-70$ 
4. Akahoshi K. Instrumentation. In: Akahoshi K, Bapaye A. Eds. Practical Handbook of Endoscopic Ultrasonography. Tokyo: Springer, 2012: 3-10.

5. Rösch T. Recent Advances Endoscopic Ultrasonography: Imaging and Beyond. Gut 2003; 52: 1220-26.

6. Wani S. Basic techniques in endoscopic ultrasoundguided fine-needle aspiration: Role of a stylet and suction. Endoscopic Ultrasound 2014; 3 (1) : 17-21.

7. Wani S, Wallace MB, Cohen J, Pike IM, Adler DG, Kochman ML, et al. Quality indicators for EUS. AJG 2015; 110: 102-13.

8. Chong AKH, Caddy GR, Desmond PV, Chen RY. Prospective study of the clinical impact of EUS. Gastrointest Endosc 2005;62: 399-405.

9. Sotoudehmanesh R, Khatibian M, Ghadir MR, Bagheri M, Hashemi-Taheri AP, Sedighi N, et al. Diagnostic accuracy of endoscopic ultrasonography In patients with inconclusive magnetic resonance imaging Diagnosis of biliopancreatic abnormalities. Indian $\mathbf{J}$. Gastroenterol 2011;30(4):156-60.

10. Bhargava SK, Usha T, Bhatt S, Kumari R, Bhargava S. Imaging in Obstructive Jaundice: A Review with Our Experience. JIMSA 2013; 26(1): 43-46.

11. ASGE guideline: the role of endoscopy in the evaluation of suspected Choledocholithiasis. Gastrointestinal Endoscopy 2010; 71(1): 1-9.
12. Rana SS, Bhasin DK, Rao C, Singh K. Role of endoscopic ultrasound in idiopathic acute pancreatitis with negative ultrasound, computed tomography, and magnetic resonance cholangiopancreatography Annals of Gastroenterology 2012; 25: 133-37.

13. Puli SR, Reddy JBK, Bechtold ML, Antillon D, Ibdah JA, Antillon MR Staging accuracy of esophageal cancer by endoscopic ultrasound: A meta-analysis and systematic review World J Gastroenterol 2008;14(10): 1479-90.

14. He LJ, Shan HB, Luo GY, Li Y, Zhang R, Gao X, et al. Endoscopic ultrasonography for staging of T1a and $\mathrm{T} 1 \mathrm{~b}$ esophageal squamous cell carcinoma. World $\mathrm{J}$ Gastroenterol 2014; 20 (5) : 1340-47.

15. Ponsaing LG, Kiss K, Loft A, Jensen LI, Hansen MB. Diagnostic procedures for submucosal tumors in the gastrointestinal tract. World J Gastroenterol; 13 (24): 3301-10.

16. Papanikolaou IS, Triantafyllou K, Kourikou A, Rösch T. Endoscopic ultrasonography for gastric submucosal lesions. World J Gastrointest Endosc. 2011; 3(5):86-94.

17. Yanai H, Noguchi $\mathrm{T}$, Mizumachi S, Tokiyamaa $\mathrm{H}$, Nakamuraa H, Tada M, et al. A blind comparison of the effectiveness of endoscopic ultrasonography and endoscopy in staging early gastric cancer. Gut 1999; 44:361-65.

18. Papanikolaoua IS, Triantafylloua M, Triantafylloua K, Rösch T. EUS in the management of gastric cancer. Ann Gastroenterol 2011; 24: 9-15. 